\title{
Some Integral Inequalities Involving Exponential Type Convex Functions and Applications
}

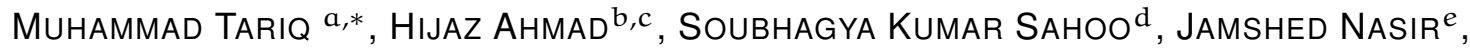 \\ SHER KHAN AWAN a \\ a Department of Basic Sciences and Related Studies, Mehran University of Engineering and \\ Technology, Jamshoro 76062, Pakistan \\ b Department of Computer Engineering, Biruni University, Istanbul 34025, Turkey \\ c Section of Mathematics, International Telematic University Uninettuno, Corso Vittorio, \\ Emanuele II, 39,00186 Roma, Italy \\ d Department of Mathematics, Institute of Technical Education and Research, Siksha O \\ Anusandhan University, Bhubaneswar 751030, Odisha, India \\ e Virtual University Islamabad, Lahore Campus, Pakistan
}

- Received: 16 August 2021 • Accepted: 22 November 2021 • Published Online: 08 December 2021

\begin{abstract}
In this present case, we focus and explore the idea of a new family of convex function namely exponential type $\mathrm{m}$-convex functions. To support this newly introduced idea, we elaborate some of its nice algebraic properties. Employing this, we investigate the novel version of Hermite-Hadamard type integral inequality. Furthermore, to enhance the paper, we present several new refinements of Hermite-Hadamard $(\mathrm{H}-\mathrm{H})$ inequality. Further, in the manner of this newly introduced idea, we investigate some applications of special means. These new results yield us some generalizations of the prior results in the literature. We believe, the methodology investigated in this paper will further inspire intrigued researchers.
\end{abstract}

Keywords: Convex function, Hölder's inequality, Power-mean integral inequality, m-type Convexity, Exponential convex function.

2010 MSC: 26A51, 26A33, 26D07, 26D10, $26 \mathrm{D} 15$.

\section{Introduction}

The theory of convexity is very important in the theoretical aspects of mathematicians and economists and also for physicists. Mathematicians use this theory, to provide the solution of problems that arise in different branches of sciences. This theory touches almost all branches of mathematics. Convex functions play an important role in many 
areas of mathematics, as well as in other areas of science, economy, engineering, medicine, industry, and business. It is especially important in the study of optimization problems, where it is distinguished by a number of convenient properties (for example, any minimum of a convex function is a global minimum, or the maximum is attained at a boundary point). This explains why there is a very rich theory of convex functions and convex sets. Optimization of convex functions has many practical applications (circuit design, controller design, modeling, etc.). Due to a lot of importance, the term "convexity" has become a rich source of inspiration and absorbing field for researchers. Interested readers are referred to $[1,2,3,4,5,6,7,8,9]$.

During the last few decades, the concept of convex analysis has played crucial and consequential role in the generalizations and extensions of theory of inequalities. Both the theory of convexity and the theory of inequality are closely related to each other. The integral inequalities have elegant and effective importance in information technology, integral operator theory, numerical integration, optimization theory, statistics, probability, and stochastic process. During the last few decades, many mathematicians and research scholars concentrated their great contributions and attentions on the study of this inequality. Thus a rich and meaningful literature on inequalities can be found for the convexity, see the references $[10,11,12,13,14,15,16,17,18]$.

\section{Preliminaries}

In this section we recall some known concepts.

Definition 2.1. [1] Let $Q: \mathbb{X} \rightarrow \mathbb{R}$ be a real valued function. A function $Q$ is said to be convex, if

$$
Q\left(d_{1} \theta+(1-\theta) d_{2}\right) \leqslant \theta Q\left(d_{1}\right)+(1-\theta) Q\left(d_{2}\right),
$$

holds for all $d_{1}, d_{2} \in \mathbb{X}$ and $\theta \in[0,1]$.

Any paper on Hermite inequalities seems to be incomplete without mentioning the wellknown Hermite-Hadamard inequality. This inequality states that, if $Q: \mathbb{X} \subset \mathbb{R} \rightarrow \mathbb{R}$ is convex in $\mathbb{X}$ for $d_{1}, d_{2} \in \mathbb{X}$ and $d_{1}<d_{2}$, then

$$
Q\left(\frac{d_{1}+d_{2}}{2}\right) \leqslant \frac{1}{d_{2}-d_{1}} \int_{d_{1}}^{d_{2}} Q(\chi) d \chi \leqslant \frac{Q\left(d_{1}\right)+Q\left(d_{2}\right)}{2} .
$$

Interested readers can refer to [19, 20, 21, 22].

The family of $m$-convex functions was first time explored and introduced by $G$. Toader in [23].

Definition 2.2. [23] A function $Q:\left[0, d_{2}\right] \rightarrow \mathbb{R}, d_{2}>0$, is said to be $m$-convex, where $\mathrm{m} \in(0,1]$, if

$$
Q\left(\theta d_{1}+m(1-\theta) d_{2}\right) \leqslant \theta Q\left(d_{1}\right)+m(1-\theta) Q\left(d_{2}\right)
$$

holds $\forall \mathrm{d}_{1}, \mathrm{~d}_{2} \in\left[0, \mathrm{~d}_{2}\right]$ and $\theta \in[0,1]$. Otherwise $\mathrm{Q}$ is $\mathrm{m}$-concave if $(-\mathrm{Q})$ is $\mathrm{m}$-convex.

Definition 2.3. [24] Let $Q$ be a nonnegative function. Then $Q: \mathbb{X} \rightarrow \mathbb{R}$, is said exponential type convex, if 


$$
Q\left(\theta d_{1}+(1-\theta) d_{2}\right) \leqslant\left(e^{\theta}-1\right) Q\left(d_{1}\right)+\left(e^{(1-\theta)}-1\right) Q\left(d_{2}\right)
$$

holds $\forall \mathrm{d}_{1}, \mathrm{~d}_{2} \in \mathbb{X}$ and $\theta \in[0,1]$.

Inspired by the above results and literatures of inequality theory, we organize the paper as follow : In section 3, we elaborate the concept and algebraic properties of exponential type $\mathrm{m}$-convex function. In section 4 , we deduce new generalization of $(\mathrm{H}-\mathrm{H})$ type inequality for the exponential type m-convex function. Next, in section 5 , we establish some refinements of the $\mathrm{H}-\mathrm{H}$ inequality, whose first derivative in absolute value at certain power are exponential type m-convex. Further, in section 6 , in the manner of this newly introduced idea, we investigate some applications of special means. Finally, in section 7 , we give a briefly conclusion.

\section{Algebraic properties of exponential type m-convex functions}

The principal focus of this section, we will present our main definition of exponential type $\mathrm{m}$-convex function and its associated properties.

Definition 3.1. Let $Q$ be a nonnegative function, then $Q: \mathbb{X} \rightarrow \mathbb{R}$, is said exponential type m-convex, if

$$
Q\left(\theta d_{1}+m(1-\theta) d_{2}\right) \leqslant\left(e^{\theta}-1\right) Q\left(d_{1}\right)+m\left(e^{(1-\theta)}-1\right) Q\left(d_{2}\right)
$$

holds $\forall \mathrm{d}_{1}, \mathrm{~d}_{2} \in \mathbb{X}, \mathrm{m} \in[0,1]$, and $\theta \in[0,1]$.

Remark 3.2. For $m=1$, we attain exponential type convexity, which is explored by İşcan in [24].

Remark 3.3. The range of the exponential type $m$-convex functions for $m \in[0,1]$ is $[0,+\infty)$.

Proof. The proof is obvious.

We explore some relations between the class exponential type m-convex functions and other class of generalized convex functions.

Lemma 3.4. The following inequalities $\left(e^{\theta}-1\right) \geqslant \theta$ and $\left(e^{(1-\theta)}-1\right) \geqslant(1-\theta)$ hold, $\forall \theta \in[0,1]$.

Proof. The proof is clearly seen and hence omitted.

Proposition 3.5. If $m \in[0,1]$, then every nonnegative $m$-convex function is exponential type m-convex function.

Proof. Since $m \in[0,1]$, and by using Lemma 3.4, we have

$$
\begin{gathered}
Q\left(\theta d_{1}+m(1-\theta) d_{2}\right) \leqslant \theta Q\left(d_{1}\right)+m(1-\theta) Q\left(d_{2}\right) \\
\leqslant\left(e^{\theta}-1\right) Q\left(d_{1}\right)+m\left(e^{(1-\theta)}-1\right) Q\left(d_{2}\right) .
\end{gathered}
$$


Theorem 3.6. Let $\mathrm{Q}, \phi:\left[\mathrm{d}_{1}, \mathrm{~d}_{2}\right] \rightarrow \mathbb{R}$. If $\mathrm{Q}$ and $\mathrm{P}$ are exponential type $\mathrm{m}$-convex functions for $m \in[0,1]$, then

1. $\mathrm{Q}+\mathrm{P}$ is exponential type $\mathrm{m}-$ convex function;

2. For nonnegative real number $\mathrm{c}, \mathrm{cQ}$ is exponential type $\mathrm{m}$-convex function.

Proof. The proof is obvious and hence omitted.

Theorem 3.7. Let $\mathrm{Q}:\left[0, \mathrm{~d}_{2}\right] \rightarrow \mathrm{J}$ be $\mathrm{m}$-convex function for $\mathrm{d}_{2}>0$ and $\mathrm{m} \in[0,1]$ and $\mathrm{Q}: \mathbb{X} \rightarrow \mathbb{R}$ is non-decreasing and exponential type $\mathrm{m}$-convex function. Then for the same fixed numbers $m \in(0,1]$, the function $\mathrm{P} \circ \mathrm{Q}:\left[0, \mathrm{~d}_{2}\right] \rightarrow \mathbb{R}$ is exponential type $\mathrm{m}$-convex.

Proof. $\forall \mathrm{d}_{1}, \mathrm{~d}_{2} \in\left[0, \mathrm{~d}_{2}\right], \mathrm{m} \in[0,1]$, and $\theta \in[0,1]$, we have

$$
\begin{gathered}
(\phi \circ Q)\left(\theta d_{1}+m(1-\theta) d_{2}\right)=P\left(Q\left(\theta d_{1}+m(1-\theta) d_{2}\right)\right) \leqslant \phi\left(\theta Q\left(d_{1}\right)+m(1-\theta) Q\left(d_{2}\right)\right) \\
\leqslant\left(e^{s \theta}-1\right)(P \circ Q)\left(d_{1}\right)+m\left(e^{(1-\theta)}-1\right)(P \circ Q)\left(d_{2}\right) .
\end{gathered}
$$

Theorem 3.8. Let $Q_{i}:\left[d_{1}, d_{2}\right] \rightarrow \mathbb{R}$ be a class of exponential type m-convex functions for $\mathrm{m} \in[0,1]$ and let $\mathrm{Q}(\mathrm{d})=\sup _{i} \mathrm{Q}_{i}(\mathrm{~d})$. If $\mathrm{E}=\left\{\mathrm{d} \in\left[\mathrm{d}_{1}, \mathrm{~d}_{2}\right]: \mathrm{Q}(\mathrm{d})<+\infty\right\} \neq \emptyset$, then $\mathrm{E}$ is an interval and $\mathrm{Q}$ is exponential type $\mathrm{m}$-convex function on $\mathrm{E}$.

Proof. For all $\mathrm{d}_{1}, \mathrm{~d}_{2} \in \mathrm{E}, \mathrm{m} \in[0,1]$, and $\theta \in[0,1]$, we have

$$
\begin{aligned}
& Q\left(\theta d_{1}+m(1-Q) d_{2}\right)=\sup _{i} Q_{i}\left(\theta d_{1}+m(1-\theta) d_{2}\right) \\
& \leqslant \sup _{i}\left[\left(e^{\theta}-1\right) Q_{i}\left(d_{1}\right)+m\left(e^{(1-\theta) s}-1\right) Q_{i}\left(d_{2}\right)\right] \\
& \leqslant\left(e^{\theta}-1\right) \sup _{i} Q_{i}\left(d_{1}\right)+m\left(e^{(1-\theta)}-1\right) \sup _{i} Q_{i}\left(d_{2}\right) \\
& =\left(e^{\theta}-1\right) Q\left(d_{1}\right)+m\left(e^{(1-\theta)}-1\right) Q\left(d_{2}\right)<+\infty .
\end{aligned}
$$

Theorem 3.9. If the function $Q:\left[\mathrm{d}_{1}, \mathrm{~d}_{2}\right] \rightarrow \mathbb{R}$ is exponential type $\mathrm{m}$-convex for $\mathrm{m} \in[0,1]$, then $\mathrm{Q}$ is bounded on $\left[\mathrm{d}_{1}, \mathrm{md}_{2}\right]$.

Proof. Suppose $x \in\left[d_{1}, d_{2}\right]$ be a point and $m \in[0,1]$ and $L=\max \left\{Q\left(d_{1}\right), m Q\left(d_{2}\right)\right\}$ and Then $\exists \theta \in[0,1]$ such that $x=\theta \mathrm{d}_{1}+\mathfrak{m}(1-\theta) \mathrm{d}_{2}$. Thus, since $e^{\theta} \leqslant e$ and $e^{(1-\theta)} \leqslant e$, we have

$$
\begin{gathered}
Q(x)=Q\left(\theta d_{1}+m(1-\theta) d_{2}\right) \leqslant\left(e^{s \theta}-1\right) Q\left(d_{1}\right)+m\left(e^{(1-\theta)}-1\right) Q\left(d_{2}\right) \\
\leqslant(e-1) L+m(e-1) L=L(m+1)(e-1)=M .
\end{gathered}
$$




\section{New generalization of $(\mathrm{H}-\mathbf{H})$ type inequality using exponential type $\mathrm{m}$-convex} function

The subject of this section is to deduce new generalizations of $(\mathrm{H}-\mathrm{H})$ type integral inequality involving exponential type $\mathrm{m}$-convex function.

Theorem 4.1. Let $Q:\left[\mathrm{d}_{1}, \mathrm{md}_{2}\right] \rightarrow \mathbb{R}$ be exponential type $\mathrm{m}$-convex function for $\mathrm{m} \in(0,1]$ and $\mathrm{d}_{1}<\mathrm{md}_{2}$. If $\mathrm{Q} \in \mathrm{L}_{1}\left(\left[\mathrm{~d}_{1}, \mathrm{md}_{2}\right]\right)$, then

$$
\begin{aligned}
\frac{1}{(\sqrt{e}-1)} Q\left(\frac{d_{1}+m d_{2}}{2}\right) \leqslant & \frac{1}{\left(m d_{2}-d_{1}\right)}\left\{\int_{d_{1}}^{m d_{2}} Q(x) d x+m \int_{\frac{d_{1}}{m}}^{d_{2}} Q(x) d x\right\} \\
& \leqslant(e-2)\left[Q\left(d_{1}\right)+Q\left(d_{2}\right)+m\left(Q\left(\frac{d_{1}}{m^{2}}\right)+Q\left(d_{2}\right)\right)\right]
\end{aligned}
$$

Proof. Let denote

$$
a_{1}=\theta d_{1}+m(1-\theta) d_{2}, \quad a_{2}=(1-\theta) \frac{d_{1}}{m}+\theta d_{2}, \quad \forall \theta \in[0,1],
$$

respectively.

Using the definition of exponential type $m$-convexity of $Q$, we have

$$
\begin{gathered}
Q\left(\frac{d_{1}+m d_{2}}{2}\right)=Q\left(\frac{a_{1}+m a_{2}}{2}\right) \\
=Q\left(\frac{\left[\theta d_{1}+m(1-\theta) d_{2}\right]+\left[(1-\theta) d_{1}+m \theta d_{2}\right]}{2}\right) \\
\leqslant(\sqrt{e}-1)\left[Q\left(\theta d_{1}+m(1-\theta) d_{2}\right)+Q\left((1-\theta) d_{1}+m \theta d_{2}\right)\right] .
\end{gathered}
$$

Now, integrating on both sides in the last inequality with respect to $\theta$ over $[0,1]$, we get

$$
\begin{gathered}
Q\left(\frac{d_{1}+m d_{2}}{2}\right) \leqslant(\sqrt{e}-1) \\
\times\left[\int_{0}^{1} Q\left(\theta d_{1}+m(1-\theta) d_{2}\right) d \theta+\int_{0}^{1} Q\left((1-\theta) \frac{d_{1}}{m}+\theta d_{2}\right) d \theta\right] \\
=\frac{(\sqrt{e}-1)}{\left(m d_{2}-d_{1}\right)}\left\{\int_{d_{1}}^{m d_{2}} Q(x) d x+m \int_{\frac{d_{1}}{m}}^{d_{2}} Q(x) d x\right\},
\end{gathered}
$$

This completes the left side inequality. For the right side inequality, using exponential type $m$-convexity of $Q$, we obtain

$$
\begin{gathered}
\frac{1}{\left(m d_{2}-d_{1}\right)}\left\{\int_{d_{1}}^{m d_{2}} Q(x) d x+m \int_{\frac{d_{1}}{m}}^{d_{2}} Q(x) d x\right\} \\
=\int_{0}^{1} Q\left(\theta d_{1}+m(1-\theta) d_{2}\right) d \theta+\int_{0}^{1} Q\left((1-\theta) \frac{d_{1}}{m}+\theta d_{2}\right) d \theta
\end{gathered}
$$




$$
\begin{aligned}
& \quad \leqslant \int_{0}^{1}\left[\left(e^{\theta}-1\right) \mathrm{Q}\left(\mathrm{d}_{1}\right)+\mathrm{m}\left(e^{(1-\theta)}-1\right) \mathrm{Q}\left(\mathrm{d}_{2}\right)\right] \mathrm{d} \theta \\
& +\int_{0}^{1}\left[\left(e^{\theta}-1\right) \mathrm{Q}\left(\mathrm{d}_{2}\right)+\mathrm{m}\left(e^{(1-\theta)}-1\right) \mathrm{Q}\left(\frac{\mathrm{d}_{1}}{\mathrm{~m}^{2}}\right)\right] \mathrm{d} \theta \\
& =(e-2)\left[\mathrm{Q}\left(\mathrm{d}_{1}\right)+\mathrm{Q}\left(\mathrm{d}_{2}\right)+\mathrm{m}\left(\mathrm{Q}\left(\frac{\mathrm{d}_{1}}{\mathrm{~m}^{2}}\right)+\mathrm{Q}\left(\mathrm{d}_{2}\right)\right)\right] .
\end{aligned}
$$

The proof is completed.

Corollary 4.2. If $\mathrm{m}=1$ in Theorem 4.1, we get (Theorem 3.1, [24]).

\section{Refinements of $(\mathrm{H}-\mathrm{H})$ type inequality via exponential type $\mathrm{m}$-convex function}

Let us establish some refinements of the $(\mathrm{H}-\mathrm{H})$ inequality for functions whose first derivative in absolute value at certain power is exponential type m-convex. First we need some new useful lemmas.

Lemma 5.1. Let $0<\mathrm{k} \leqslant 1$ and a mapping $\mathrm{Q}:\left[\mathrm{d}_{1}, \frac{\mathrm{d}_{2}}{\mathrm{k}}\right] \rightarrow \mathbb{R}$ is differentiable on $\left(\mathrm{d}_{1}, \frac{\mathrm{d}_{2}}{\mathrm{k}}\right)$ with $0<\mathrm{d}_{1}<\mathrm{d}_{2}$. If $\mathrm{Q}^{\prime} \in \mathrm{L}_{1}\left[\mathrm{~d}_{1}, \frac{\mathrm{d}_{2}}{\mathrm{k}}\right]$ and $\mathrm{m} \in[0,1]$, then

$$
\begin{gathered}
\frac{Q\left(d_{1}\right)+Q\left(\frac{m d_{2}}{k}\right)}{2}-\frac{k}{m d_{2}-k d_{1}} \int_{d_{1}}^{\frac{m d_{2}}{k}} Q(\theta) d \theta=\left(\frac{m d_{2}-k d_{1}}{2 k}\right) \\
\quad \times \int_{0}^{1}(1-2 \theta) Q^{\prime}\left(\theta d_{1}+m(1-\theta) \frac{d_{2}}{k}\right) d \theta .
\end{gathered}
$$

Proof. Using the integrating by parts, we have

$$
\begin{aligned}
& \left(\frac{m d_{2}-k d_{1}}{2 k}\right) \int_{0}^{1}(1-2 \theta) Q^{\prime}\left(\theta d_{1}+m(1-\theta) \frac{d_{2}}{k}\right) d \theta \\
& =\left(\frac{m d_{2}-k d_{1}}{2 k}\right)\left\{\left.\frac{(1-2 \theta) Q\left(\theta d_{1}+m(1-\theta) \frac{d_{2}}{k}\right)}{d_{1}-\frac{m d_{2}}{k}}\right|_{0} ^{1}-\int_{0}^{1} \frac{Q\left(\theta d_{1}+m(1-\theta) \frac{d_{2}}{k}\right)}{d_{1}-\frac{m d_{2}}{k}}(-2) d \theta\right\} \\
& =\left(\frac{m d_{2}-k d_{1}}{2 k}\right)\left\{\frac{-Q\left(d_{1}\right)-Q\left(\frac{m d_{2}}{k}\right)}{\frac{k d_{1}-m d_{2}}{k}}+\frac{2 k}{k d_{1}-m d_{2}} \int_{0}^{1} Q\left(\theta d_{1}+m(1-\theta) \frac{d_{2}}{k}\right) d \theta\right\} \\
& =\left(\frac{m d_{2}-k d_{1}}{2 k}\right)\left\{\frac{k\left(Q\left(d_{1}\right)+Q\left(\frac{m d_{2}}{k}\right)\right)}{m d_{2}-k d_{1}}-\frac{2 k}{m d_{2}-k d_{1}} \int_{0}^{1} Q\left(\theta d_{1}+m(1-\theta) \frac{d_{2}}{k}\right) d \theta\right\} \\
& =\frac{Q\left(d_{1}\right)+Q\left(\frac{m d_{2}}{k}\right)}{2}-\frac{k}{m d_{2}-k d_{1}} \int_{d_{1}}^{\frac{m d_{2}}{k}} Q(d) d \theta .
\end{aligned}
$$

This completes the proof. 
Remark 5.2. If $m=1$ in Lemma 5.1, we have

$$
\begin{gathered}
\frac{Q\left(d_{1}\right)+Q\left(\frac{d_{2}}{k}\right)}{2}-\frac{k}{d_{2}-k d_{1}} \int_{d_{1}}^{\frac{d_{2}}{k}} Q(\theta) d \theta=\left(\frac{d_{2}-k d_{1}}{2 k}\right) \\
\times \int_{0}^{1}(1-2 \theta) Q^{\prime}\left(\theta d_{1}+(1-\theta) \frac{d_{2}}{k}\right) d \theta .
\end{gathered}
$$

Remark 5.3. If $k=1$ in Lemma 5.1, we have

$$
\begin{gathered}
\frac{Q\left(d_{1}\right)+Q\left(m d_{2}\right)}{2}-\frac{1}{m d_{2}-d_{1}} \int_{d_{1}}^{m d_{2}} Q(\theta) d \theta=\left(\frac{m d_{2}-d_{1}}{2}\right) \\
\times \int_{0}^{1}(1-2 \theta) Q^{\prime}\left(\theta d_{1}+m(1-\theta) d_{2}\right) d \theta .
\end{gathered}
$$

Remark 5.4. If $m=k=1$ in Lemma 5.1, then we have a Lemma 2.1 in [25].

Lemma 5.5. Let $0<\mathrm{k} \leqslant 1$ and $\mathrm{Q}:\left[\mathrm{kd}_{1}, \mathrm{~d}_{2}\right] \rightarrow \mathbb{R}$ is differentiable on $\left(\mathrm{kd}_{1}, \mathrm{~d}_{2}\right)$ with $0<\mathrm{d}_{1}<\mathrm{d}_{2}$. If $\mathrm{Q}^{\prime} \in \mathrm{L}_{1}\left[\mathrm{kd}_{1}, \mathrm{a}_{2}\right]$ and $\mathrm{m} \in[0,1]$, then

$$
\begin{gathered}
\frac{Q\left(m k d_{1}\right)+Q\left(d_{2}\right)}{2}-\frac{1}{d_{2}-k d_{1}} \int_{m k d_{1}}^{d_{2}} Q(\theta) d \theta=\left(\frac{d_{2}-m k d_{1}}{2}\right) \\
\times \int_{0}^{1}(2 \theta-1) Q^{\prime}\left(\theta d_{2}+m k(1-\theta) d_{1}\right) d \theta .
\end{gathered}
$$

Proof. Using the integrating by parts, we have

$$
\begin{aligned}
& \left(\frac{d_{2}-m k d_{1}}{2}\right) \int_{0}^{1}(2 \theta-1) Q^{\prime}\left(\theta d_{2}+m k(1-\theta) d_{1}\right) \\
& =\left(\frac{d_{2}-m k d_{1}}{2}\right) \\
& \times\left\{\left.\frac{(2 \theta-1) Q\left(\theta d_{2}+m k(1-\theta) d_{1}\right)}{d_{2}-m k d_{1}}\right|_{0} ^{1}-\int_{0}^{1} \frac{Q\left(\theta d_{2}+m k(1-\theta) d_{1}\right)}{d_{2}-m k d_{1}}(2) d \theta\right\} \\
& =\left(\frac{d_{2}-m k d_{1}}{2}\right)\left\{\frac{Q\left(d_{2}\right)+Q\left(m k d_{1}\right)}{d_{2}-m k d_{1}}-\frac{2}{d_{2}-m k d_{1}} \int_{0}^{1} Q\left(\theta d_{2}+m k(1-\theta) d_{1}\right) d \theta\right\} \\
& =\left(\frac{d_{2}-m k d_{1}}{2}\right)\left\{\frac{Q\left(d_{2}\right)+Q\left(m k d_{1}\right)}{d_{2}-m k d_{1}}-\frac{2}{d_{2}-k d_{1}} \int_{0}^{1} Q\left(\theta d_{2}+m k(1-\theta) d_{1}\right) d \theta\right\} \\
& =\frac{Q\left(m k d_{1}\right)+Q\left(d_{2}\right)}{2}-\frac{1}{d_{2}-m k d_{1}} \int_{m k d_{1}}^{d_{2}} Q(\theta) d \theta,
\end{aligned}
$$

which completes the proof. 
Remark 5.6. If $m=1$ in Lemma 5.5, we have

$$
\begin{gathered}
\frac{Q\left(k d_{1}\right)+Q\left(d_{2}\right)}{2}-\frac{1}{d_{2}-k d_{1}} \int_{k d_{1}}^{d_{2}} Q(\theta) d \theta=\left(\frac{d_{2}-k d_{1}}{2}\right) \\
\times \int_{0}^{1}(2 \theta-1) Q^{\prime}\left(\theta d_{2}+k(1-\theta) d_{1}\right) d \theta
\end{gathered}
$$

Remark 5.7. If $k=1$ in Lemma 5.5, we have

$$
\begin{gathered}
\frac{Q\left(m d_{1}\right)+Q\left(d_{2}\right)}{2}-\frac{1}{d_{2}-d_{1}} \int_{m d_{1}}^{d_{2}} Q(\theta) d \theta=\left(\frac{d_{2}-m d_{1}}{2}\right) \\
\times \int_{0}^{1}(2 \theta-1) Q^{\prime}\left(\theta d_{2}+m(1-\theta) d_{1}\right) d \theta .
\end{gathered}
$$

Remark 5.8. If $m=k=1$ in Lemma 5.5, then we have a Lemma 2.1 in [25].

Theorem 5.9. Let $\mathrm{Q}: \mathbb{X} \rightarrow \mathbb{R}$ is differentiable on $\mathbb{X}$ with $0<\mathrm{d}_{1}<\mathrm{d}_{2}$. and $0<\mathrm{k} \leqslant 1$. If $\left|\mathrm{Q}^{\prime}\right|^{\mathrm{q}}$ is exponential type $\mathrm{m}$-convex function on $\mathbb{X}$ for $\mathrm{q}>1$ and $\frac{1}{\mathrm{q}}+\frac{1}{\mathrm{p}}=1$, then for $\mathrm{m} \in[0,1]$, the following inequality holds:

$$
\begin{aligned}
& \left|\frac{Q\left(d_{1}\right)+Q\left(\frac{m d_{2}}{k}\right)}{2}-\frac{k}{m d_{2}-k d_{1}} \int_{d_{1}}^{\frac{m d_{2}}{k}} Q(\theta) d \theta\right| \leqslant\left(\frac{m d_{2}-k d_{1}}{2 k}\right)\left(\frac{1}{p+1}\right)^{\frac{1}{p}} \\
& \times\left\{(e-2)\left(\left|Q^{\prime}\left(d_{1}\right)\right|^{q}+m\left|Q^{\prime}\left(\frac{d_{2}}{k}\right)\right|^{q}\right)\right\}^{\frac{1}{q}} .
\end{aligned}
$$

Proof. From Lemma 5.1, Hölder's inequality and exponential type m-convexity of $\left|Q^{\prime}\right|^{q}$, we have

$$
\begin{aligned}
& \left|\frac{Q\left(d_{1}\right)+Q\left(\frac{m d_{2}}{k}\right)}{2}-\frac{k}{m d_{2}-k d_{1}} \int_{d_{1}}^{\frac{m d_{2}}{k}} Q(\theta) d \theta\right| \\
& \leqslant\left(\frac{m d_{2}-k d_{1}}{2 k}\right)\left(\int_{0}^{1}|1-2 \theta|^{p} d \theta\right)^{\frac{1}{p}}\left\{\int_{0}^{1}\left|Q^{\prime}\left(\theta d_{1}+m(1-\theta) \frac{d_{2}}{k}\right)\right|^{q} d \theta\right\}^{\frac{1}{q}} \\
& \leqslant\left(\frac{m d_{2}-k d_{1}}{2 k}\right)\left(\int_{0}^{1}|1-2 \theta|^{p} d \theta\right)^{\frac{1}{p}} \\
& \times\left\{\int_{0}^{1}\left[\left(e^{\theta}-1\right)\left|Q^{\prime}\left(d_{1}\right)\right|^{q}+m\left(e^{(1-\theta)}-1\right)\left|Q^{\prime}\left(\frac{d_{2}}{k}\right)\right|^{q}\right] d \theta\right\}^{\frac{1}{q}} \\
& =\left(\frac{m d_{2}-k d_{1}}{2 k}\right)\left(\frac{1}{p+1}\right)^{\frac{1}{p}}\left\{(e-2)\left(\left|Q^{\prime}\left(d_{1}\right)\right|^{q}+m\left|Q^{\prime}\left(\frac{d_{2}}{k}\right)\right|^{q}\right)\right\}^{\frac{1}{q}} .
\end{aligned}
$$

This completes the proof. 
Remark 5.10. If $m=1$ in Theorem 5.9, we have

$$
\begin{aligned}
& \left|\frac{Q\left(d_{1}\right)+Q\left(\frac{d_{2}}{k}\right)}{2}-\frac{k}{d_{2}-k d_{1}} \int_{d_{1}}^{\frac{d_{2}}{k}} Q(\theta) d \theta\right| \leqslant\left(\frac{d_{2}-k d_{1}}{2 k}\right)\left(\frac{1}{p+1}\right)^{\frac{1}{p}} \\
& \times\left\{(e-2)\left(\left|Q^{\prime}\left(d_{1}\right)\right|^{q}+\left|Q^{\prime}\left(\frac{d_{2}}{k}\right)\right|^{q}\right)\right\}^{\frac{1}{q}} .
\end{aligned}
$$

Remark 5.11. If $k=1$ in Theorem 5.9, we have

$$
\begin{aligned}
& \left|\frac{Q\left(d_{1}\right)+Q\left(m d_{2}\right)}{2}-\frac{1}{m d_{2}-d_{1}} \int_{d_{1}}^{m d_{2}} Q(\theta) d \theta\right| \leqslant\left(\frac{m d_{2}-d_{1}}{2}\right)\left(\frac{1}{p+1}\right)^{\frac{1}{p}} \\
& \times\left\{(e-2)\left(\left|Q^{\prime}\left(d_{1}\right)\right|^{q}+m\left|Q^{\prime}\left(d_{2}\right)\right|^{q}\right)\right\}^{\frac{1}{q}} .
\end{aligned}
$$

Remark 5.12. If $m=k=1$ in Theorem 5.9, we have

$$
\begin{aligned}
& \left|\frac{Q\left(d_{1}\right)+Q\left(d_{2}\right)}{2}-\frac{1}{d_{2}-d_{1}} \int_{d_{1}}^{d_{2}} Q(\theta) d \theta\right| \leqslant\left(\frac{d_{2}-d_{1}}{2}\right)\left(\frac{1}{p+1}\right)^{\frac{1}{p}} \\
& \times\left\{(e-2)\left(\left|Q^{\prime}\left(d_{1}\right)\right|^{q}+\left|Q^{\prime}\left(d_{2}\right)\right|^{q}\right)\right\}^{\frac{1}{q}} .
\end{aligned}
$$

Theorem 5.13. Let $\mathrm{Q}: \mathbb{X} \rightarrow \mathbb{R}$ is differentiable on $\mathbb{X}$ with $0<\mathrm{d}_{1}<\mathrm{d}_{2}$. and $0<\mathrm{k} \leqslant 1$. If $\left|\mathrm{Q}^{\prime}\right|^{\mathrm{q}}$ is exponential type $\mathrm{m}$-convex function on $\mathbb{X}$ for $\mathrm{q} \geqslant 1$, and $\mathrm{m} \in[0,1]$, we have

$$
\begin{aligned}
& \left|\frac{\mathrm{Q}\left(\mathrm{d}_{1}\right)+\mathrm{Q}\left(\frac{\mathrm{md_{2 }}}{\mathrm{k}}\right)}{2}-\frac{\mathrm{k}}{\mathrm{md}_{2}-k \mathrm{~d}_{1}} \int_{\mathrm{d}_{1}}^{\frac{m \mathrm{~d}_{2}}{\mathrm{k}}} \mathrm{Q}(\theta) \mathrm{d} \theta\right| \leqslant\left(\frac{m \mathrm{~d}_{2}-k \mathrm{~d}_{1}}{2 k}\right)\left(\frac{1}{2}\right)^{1-\frac{1}{q}} \\
& \times\left\{\left(\frac{8 \sqrt{e}-2 e-7}{2}\right)\left(\left|\mathrm{Q}^{\prime}\left(\mathrm{d}_{1}\right)\right|^{\mathrm{q}}+\mathrm{m}\left|\mathrm{Q}^{\prime}\left(\frac{\mathrm{d}_{2}}{\mathrm{k}}\right)\right|^{\mathrm{q}}\right)\right\}^{\frac{1}{q}} .
\end{aligned}
$$

Proof. From Lemma 5.1, power mean inequality and exponential type m-convexity of 


$$
\begin{aligned}
& \left|Q^{\prime}\right|^{q} \text {, we have } \\
& \left|\frac{Q\left(d_{1}\right)+Q\left(\frac{m d_{2}}{k}\right)}{2}-\frac{k}{m d_{2}-k d_{1}} \int_{d_{1}}^{\frac{m d_{2}}{k}} Q(\theta) d \theta\right| \\
& \leqslant\left(\frac{m d_{2}-k d_{1}}{2 k}\right)\left\{\int_{0}^{1}|1-2 \theta|\left|Q^{\prime}\left(\theta d_{1}+m(1-\theta) \frac{d_{2}}{k}\right)\right| d \theta\right\} \\
& \leqslant\left(\frac{m d_{2}-k d_{1}}{2 k}\right)\left(\int_{0}^{1}|1-2 \theta| d \theta\right)^{1-\frac{1}{q}}\left\{\int_{0}^{1}|1-2 \theta|\left|Q^{\prime}\left(\theta d_{1}+m(1-\theta) \frac{d_{2}}{k}\right)\right|^{q} d \theta\right\}^{\frac{1}{q}} \\
& \leqslant\left(\frac{\mathrm{md}_{2}-k \mathrm{~d}_{1}}{2 k}\right)\left(\int_{0}^{1}|1-2 \theta| \mathrm{d} \theta\right)^{1-\frac{1}{q}} \\
& \times\left\{\int_{0}^{1}|1-2 \theta|\left[\left(e^{\theta}-1\right)\left|\mathrm{Q}^{\prime}\left(\mathrm{d}_{1}\right)\right|^{\mathrm{q}}+\mathrm{m}\left(e^{(1-\theta)}-1\right)\left|\mathrm{Q}^{\prime}\left(\frac{\mathrm{d}_{2}}{\mathrm{k}}\right)\right|^{\mathrm{q}}\right] \mathrm{d} \theta\right\}^{\frac{1}{q}} \\
& =\left(\frac{m d_{2}-k d_{1}}{2 k}\right)\left(\frac{1}{2}\right)^{1-\frac{1}{q}} \\
& \times\left\{\left(\frac{8 \sqrt{e}-2 e-7}{2}\right)\left(\left|\mathrm{Q}^{\prime}\left(\mathrm{d}_{1}\right)\right|^{\mathrm{q}}+\mathrm{m}\left|\mathrm{Q}^{\prime}\left(\frac{\mathrm{d}_{2}}{\mathrm{k}}\right)\right|^{\mathrm{q}}\right)\right\}^{\frac{1}{\mathrm{q}}} \text {. }
\end{aligned}
$$

This completes the proof.

Remark 5.14. If $m=1$ in Theorem 5.13, we get

$$
\begin{aligned}
& \left|\frac{Q\left(d_{1}\right)+Q\left(\frac{d_{2}}{k}\right)}{2}-\frac{k}{d_{2}-k d_{1}} \int_{d_{1}}^{\frac{d_{2}}{k}} Q(\theta) d \theta\right| \leqslant\left(\frac{d_{2}-k d_{1}}{2 k}\right)\left(\frac{1}{2}\right)^{1-\frac{1}{q}} \\
& \times\left\{\left(\frac{8 \sqrt{e}-2 e-7}{2}\right)\left(\left|Q^{\prime}\left(d_{1}\right)\right|^{q}+\left|Q^{\prime}\left(\frac{d_{2}}{k}\right)\right|^{q}\right)\right\}^{\frac{1}{q}} .
\end{aligned}
$$

Remark 5.15. If $k=1$ in Theorem 5.13, we get

$$
\begin{aligned}
& \left|\frac{Q\left(d_{1}\right)+Q\left(m d_{2}\right)}{2}-\frac{1}{m d_{2}-d_{1}} \int_{d_{1}}^{m d_{2}} Q(\theta) d \theta\right| \leqslant\left(\frac{m d_{2}-d_{1}}{2}\right)\left(\frac{1}{2}\right)^{1-\frac{1}{q}} \\
& \times\left\{\left(\frac{8 \sqrt{e}-2 e-7}{2}\right)\left(\left|Q^{\prime}\left(d_{1}\right)\right|^{q}+m\left|Q^{\prime}\left(d_{2}\right)\right|^{q}\right)\right\}^{\frac{1}{q}} .
\end{aligned}
$$

Remark 5.16. If $m=k=1$ in Theorem 5.13, we get

$$
\begin{aligned}
& \left|\frac{Q\left(d_{1}\right)+Q\left(d_{2}\right)}{2}-\frac{1}{d_{2}-d_{1}} \int_{d_{1}}^{d_{2}} Q(\theta) d \theta\right| \leqslant\left(\frac{d_{2}-d_{1}}{2}\right)\left(\frac{1}{2}\right)^{1-\frac{1}{q}} \\
& \times\left\{\left(\frac{8 \sqrt{e}-2 e-7}{2}\right)\left(\left|Q^{\prime}\left(d_{1}\right)\right|^{q}+\left|Q^{\prime}\left(d_{2}\right)\right|^{q}\right)\right\}^{\frac{1}{q}} .
\end{aligned}
$$


Theorem 5.17. Let $\mathrm{Q}: \mathbb{X} \rightarrow \mathbb{R}$ is differentiable on $\mathbb{X}$ with $0<\mathrm{d}_{1}<\mathrm{d}_{2}$. and $0<\mathrm{k} \leqslant 1$. If $\left|\mathrm{Q}^{\prime}\right|^{\mathrm{q}}$ is exponential type $\mathrm{m}$-convex function on $\mathbb{X}$ for $\mathrm{q}>1$ and $\frac{1}{\mathrm{q}}+\frac{1}{\mathrm{p}}=1$, then for $\mathrm{m} \in[0,1]$, we have:

$$
\begin{aligned}
& \left|\frac{Q\left(m k d_{1}\right)+Q\left(d_{2}\right)}{2}-\frac{1}{d_{2}-m k d_{1}} \int_{m k d_{1}}^{d_{2}} Q(\theta) d \theta\right| \leqslant\left(\frac{d_{2}-m k d_{1}}{2}\right)\left(\frac{1}{p+1}\right)^{\frac{1}{p}} \\
& \times\left\{(e-2)\left(m\left|Q^{\prime}\left(k d_{1}\right)\right|^{q}+\left|Q^{\prime}\left(d_{2}\right)\right|^{q}\right)\right\}^{\frac{1}{q}} .
\end{aligned}
$$

Proof. From Lemma 5.5, Hölder's inequality and exponential type m-convexity of $\left|Q^{\prime}\right|^{q}$, we have

$$
\begin{aligned}
& \left|\frac{Q\left(k d_{1}\right)+Q\left(d_{2}\right)}{2}-\frac{1}{d_{2}-k d_{1}} \int_{k d_{1}}^{d_{2}} Q(\theta) d \theta\right| \\
& \leqslant\left(\frac{d_{2}-k d_{1}}{2}\right)\left(\int_{0}^{1}|2 \theta-1|^{p} d \theta\right)^{\frac{1}{p}}\left\{\int_{0}^{1}\left|Q^{\prime}\left(\theta d_{2}+m k(1-\theta) d_{1}\right)\right|^{q} d \theta\right\}^{\frac{1}{q}} \\
& \leqslant\left(\frac{d_{2}-m k d_{1}}{2}\right)\left(\int_{0}^{1}|2 \theta-1|^{p} d \theta\right)^{\frac{1}{p}} \\
& \times\left\{\int_{0}^{1}\left[\left(e^{\theta}-1\right)\left|Q^{\prime}\left(d_{2}\right)\right|^{q}+m\left(e^{(1-\theta)}-1\right)\left|Q^{\prime}\left(k d_{1}\right)\right|^{q}\right] d \theta\right\}^{\frac{1}{q}} \\
& =\left(\frac{d_{2}-m k d_{1}}{2}\right)\left(\frac{1}{p+1}\right)^{\frac{1}{p}} \\
& \times\left\{(e-2)\left(m\left|Q^{\prime}\left(k d_{1}\right)\right|^{q}+\left|Q^{\prime}\left(d_{2}\right)\right|^{q}\right)\right\}^{\frac{1}{q}} .
\end{aligned}
$$

This completes the proof.

Remark 5.18. If $m=1$ in Theorem 5.17, we obtain

$$
\begin{aligned}
& \left|\frac{\mathrm{Q}\left(k d_{1}\right)+\mathrm{Q}\left(\mathrm{d}_{2}\right)}{2}-\frac{1}{\mathrm{~d}_{2}-k \mathrm{~d}_{1}} \int_{k d_{1}}^{\mathrm{d}_{2}} \mathrm{Q}(\theta) \mathrm{d} \theta\right| \leqslant\left(\frac{\mathrm{d}_{2}-k \mathrm{~d}_{1}}{2}\right)\left(\frac{1}{p+1}\right)^{\frac{1}{p}} \\
& \times\left\{(e-2)\left(\left|\mathrm{Q}^{\prime}\left(k \mathrm{~d}_{1}\right)\right|^{\mathrm{q}}+\left|\mathrm{Q}^{\prime}\left(\mathrm{d}_{2}\right)\right|^{\mathrm{q}}\right)\right\}^{\frac{1}{q}} .
\end{aligned}
$$

Remark 5.19. If $k=1$ in Theorem 5.17, we obtain

$$
\begin{aligned}
& \left|\frac{Q\left(m d_{1}\right)+Q\left(d_{2}\right)}{2}-\frac{1}{d_{2}-m d_{1}} \int_{m d_{1}}^{d_{2}} Q(\theta) d \theta\right| \leqslant\left(\frac{d_{2}-m d_{1}}{2}\right)\left(\frac{1}{p+1}\right)^{\frac{1}{p}} \\
& \times\left\{(e-2)\left(m\left|Q^{\prime}\left(d_{1}\right)\right|^{q}+\left|Q^{\prime}\left(d_{2}\right)\right|^{q}\right)\right\}^{\frac{1}{q}} .
\end{aligned}
$$


Remark 5.20. If $m=k=1$ in Theorem 5.17, we obtain

$$
\begin{aligned}
& \left|\frac{Q\left(d_{1}\right)+Q\left(d_{2}\right)}{2}-\frac{1}{d_{2}-d_{1}} \int_{d_{1}}^{d_{2}} Q(\theta) d \theta\right| \leqslant\left(\frac{d_{2}-d_{1}}{2}\right)\left(\frac{1}{p+1}\right)^{\frac{1}{p}} \\
& \times\left\{(e-2)\left(\left|Q^{\prime}\left(d_{1}\right)\right|^{q}+\left|Q^{\prime}\left(d_{2}\right)\right|^{q}\right)\right\}^{\frac{1}{q}} .
\end{aligned}
$$

Theorem 5.21. Let $\mathrm{Q}: \mathbb{X} \rightarrow \mathbb{R}$ is differentiable on $\mathbb{X}$ with $0<\mathrm{d}_{1}<\mathrm{d}_{2}$. and $0<\mathrm{k} \leqslant 1$. If $\left|\mathrm{Q}^{\prime}\right|^{\mathrm{q}}$ is exponential type $\mathrm{m}$-convex function on $\mathbb{X}$ for $\mathrm{q} \geqslant 1$, and $\mathrm{m} \in[0,1]$, then we have:

$$
\begin{aligned}
& \left|\frac{Q\left(m k d_{1}\right)+Q\left(d_{2}\right)}{2}-\frac{1}{d_{2}-m k d_{1}} \int_{m k d_{1}}^{d_{2}} Q(\theta) d \theta\right| \leqslant\left(\frac{d_{2}-k d_{1}}{2}\right)\left(\frac{1}{2}\right)^{1-\frac{1}{q}} \\
& \times\left\{\frac{8 \sqrt{e}-2 e-7}{2}\left(m\left|Q^{\prime}\left(k d_{1}\right)\right|^{q}+\left|Q^{\prime}\left(d_{2}\right)\right|^{q}\right)\right\}^{\frac{1}{q}},
\end{aligned}
$$

Proof. From Lemma 5.5, power mean inequality and exponential type m-convexity of $\left|Q^{\prime}\right|^{q}$, we have

$$
\begin{aligned}
& \left|\frac{Q\left(k d_{1}\right)+Q\left(d_{2}\right)}{2}-\frac{1}{d_{2}-k d_{1}} \int_{k d_{1}}^{d_{2}} Q(\theta) d \theta\right| \\
& \leqslant\left(\frac{d_{2}-m k d_{1}}{2}\right)\left\{\int_{0}^{1}|2 \theta-1|\left|Q^{\prime}\left(\theta d_{2}+m k(1-\theta) d_{1}\right)\right| d \theta\right\} \\
& \leqslant\left(\frac{d_{2}-m k d_{1}}{2}\right)\left(\int_{0}^{1}|2 \theta-1| d \theta\right)^{1-\frac{1}{q}} \\
& \times\left\{\int_{0}^{1}|2 \theta-1|\left|Q^{\prime}\left(\theta d_{2}+m k(1-\theta) d_{1}\right)\right|^{q} d \theta\right\}^{\frac{1}{q}} \\
& \leqslant\left(\frac{d_{2}-m k d_{1}}{2}\right)\left(\int_{0}^{1}|2 \theta-1| d \theta\right)^{1-\frac{1}{q}} \\
& \times\left[\int_{0}^{1}|2 \theta-1|\left\{m\left(e^{(1-\theta)}-1\right)\left|Q^{\prime}\left(k d_{1}\right)\right|^{q}+\left(e^{\theta}-1\right)\left|Q^{\prime}\left(d_{2}\right)\right|^{q}\right\} d \theta\right]^{\frac{1}{q}} \\
& =\left(\frac{d_{2}-m k d_{1}}{2}\right)\left(\frac{1}{2}\right)^{1-\frac{1}{q}} \\
& \times\left\{\left(\frac{8 \sqrt{e}-2 e-7}{2}\right)\left(m\left|Q^{\prime}\left(k d_{1}\right)\right|^{q}+\left|Q^{\prime}\left(d_{2}\right)\right|^{q}\right)\right\}^{\frac{1}{q}} \cdot
\end{aligned}
$$

This completes the proof. 
Remark 5.22. If $m=1$ in Theorem 5.21, we have

$$
\begin{aligned}
& \left|\frac{\mathrm{Q}\left(k \mathrm{~d}_{1}\right)+\mathrm{Q}\left(\mathrm{d}_{2}\right)}{2}-\frac{1}{\mathrm{~d}_{2}-k \mathrm{~d}_{1}} \int_{k \mathrm{~d}_{1}}^{\mathrm{d}_{2}} \mathrm{Q}(\theta) \mathrm{d} \theta\right| \leqslant\left(\frac{\mathrm{d}_{2}-k \mathrm{~d}_{1}}{2}\right)\left(\frac{1}{2}\right)^{1-\frac{1}{q}} \\
& \times\left\{\frac{8 \sqrt{e}-2 e-7}{2}\left(\left|\mathrm{Q}^{\prime}\left(k \mathrm{~d}_{1}\right)\right|^{\mathrm{q}}+\left|\mathrm{Q}^{\prime}\left(\mathrm{d}_{2}\right)\right|^{\mathrm{q}}\right)\right\}^{\frac{1}{q}} .
\end{aligned}
$$

Remark 5.23. If $k=1$ in Theorem 5.21, we have

$$
\begin{aligned}
& \left|\frac{Q\left(m d_{1}\right)+Q\left(d_{2}\right)}{2}-\frac{1}{d_{2}-m d_{1}} \int_{m d_{1}}^{d_{2}} Q(\theta) d \theta\right| \leqslant\left(\frac{d_{2}-d_{1}}{2}\right)\left(\frac{1}{2}\right)^{1-\frac{1}{q}} \\
& \times\left\{\frac{8 \sqrt{e}-2 e-7}{2}\left(m\left|Q^{\prime}\left(d_{1}\right)\right|^{q}+\left|Q^{\prime}\left(d_{2}\right)\right|^{q}\right)\right\}^{\frac{1}{q}} .
\end{aligned}
$$

Remark 5.24. If $\mathrm{m}=\mathrm{k}=1$ in Theorem 5.21, we have

$$
\begin{aligned}
& \left|\frac{Q\left(d_{1}\right)+Q\left(d_{2}\right)}{2}-\frac{1}{d_{2}-d_{1}} \int_{d_{1}}^{d_{2}} Q(\theta) d \theta\right| \leqslant\left(\frac{d_{2}-d_{1}}{2}\right)\left(\frac{1}{2}\right)^{1-\frac{1}{q}} \\
& \times\left\{\frac{8 \sqrt{e}-2 e-7}{2}\left(\left|Q^{\prime}\left(d_{1}\right)\right|^{q}+\left|Q^{\prime}\left(d_{2}\right)\right|^{q}\right)\right\}^{\frac{1}{q}} .
\end{aligned}
$$

\section{Applications}

In this section, we recall the following special means of two positive numbers $d_{1}, d_{2}$ with $\mathrm{d}_{1}<\mathrm{d}_{2}$ :

(1) The arithmetic mean

$$
A=A\left(d_{1}, d_{2}\right)=\frac{d_{1}+d_{2}}{2}, \quad d_{1}, d_{2} \geqslant 0 .
$$

(2) The geometric mean

$$
G=G\left(d_{1}, d_{2}\right)=\sqrt{d_{1} d_{2}}, \quad d_{1}, d_{2} \geqslant 0
$$

(3) The harmonic mean

$$
H=H\left(d_{1}, d_{2}\right)=\frac{2 d_{1} d_{2}}{d_{1}+d_{2}}, \quad d_{1}, d_{2}>0 .
$$

(4) The logarithmic mean

$$
L=L\left(d_{1}, d_{2}\right)=\left\{\begin{array}{l}
\frac{d_{2}-d_{1}}{\ln d_{2}-\ln d_{1}}, \quad d_{1} \neq d_{2}, \\
d_{1}, \quad d_{1}=d_{2} ;
\end{array} \quad d_{1}, d_{2}>0 .\right.
$$

The following relationship are well-known in the literature.

$$
H\left(d_{1}, d_{2}\right) \leqslant G\left(d_{1}, d_{2}\right) \leqslant A\left(d_{1}, d_{2}\right) .
$$


Proposition 6.1. Let $\mathrm{d}_{1}, \mathrm{~d}_{2} \in[0, \infty)$ with $\mathrm{d}_{1}<\mathrm{d}_{2}$ and $\mathrm{r} \in(-\infty, 0) \cup[1, \infty) \backslash\{-1\}$. Then the inequalities

$$
\frac{A^{r}\left(d_{1}, d_{2}\right)}{2(\sqrt{e}-1)} \leqslant L_{r}^{r}\left(d_{1}, d_{2}\right) \leqslant 2(e-2) A\left(d_{1}^{r}, d_{2}^{r}\right)
$$

holds.

Proof. If we put $\mathrm{Q}(x)=x^{\mathrm{r}}, x \in[0, \infty)$ and $\mathrm{m}=1$ in the above theorem 4.1, then we can easily obtained the inequality 6.1 .

Proposition 6.2. Let $\mathrm{d}_{1}, \mathrm{~d}_{2} \in(0, \infty)$ with $\mathrm{d}_{1}<\mathrm{d}_{2}$. Then the inequalities

$$
\frac{A^{r}\left(d_{1}, d_{2}\right)}{2(\sqrt{e}-1)} \leqslant L^{-1}\left(d_{1}, d_{2}\right) \leqslant 2(e-2) H^{-1}\left(d_{1}, d_{2}\right),
$$

holds.

Proof. If we put $\mathrm{Q}(x)=x^{-1}, \quad x \in(0, \infty)$ and $\mathrm{m}=1$ in the above theorem 4.1 , then we can easily obtained the inequality 6.2 .

\section{Conclusion}

In this paper, authors presented new assessment of $(\mathrm{H}-\mathrm{H})$ type inequalities for a new type of convexity, called exponential type m-convex function. We have achieved refinements of the $(\mathrm{H}-\mathrm{H})$ inequality for functions whose first derivative in absolute value at certain power are exponential type m-convex. As of late, numerous mathematicians put exertion into the hypothesis of inequality to express new dimension to mathematical analysis. Because of widespread perspectives and significance, this hypothesis has become an alluring and engrossing field for researchers. We trust that our novel thoughts and strategies may propel numerous scientists in this intriguing field.

\section{References}

[1] Niculescu CP and Persson LE (2006). Convex functions and their applications. Springer, New York.

[2] Özdemir ME, Yildiz C, Akdemir AO and E. Set (2013). On some inequalities for s-convex functions and applications. J. Inequal. Appl. 333, 2-11. https://doi.org/10.1186/1029-242X-2013-333

[3] Butt SI, Rashid S, Tariq M and Wang XH (2021). Novel refinements via n-polynomial harmonically stype convex functions and Applications in special functions. J. Funt, Spaces. 1-17. https://doi.org/ $10.1155 / 2021 / 6615948$

[4] Xi BY and Qi F (2012). Some integral inequalities of Hermite-Hadamard type for convex functions with applications to means. J. Funct. Spaces Appl. (2012), Art. ID 980438, 1-14. https://doi.org/10.1155/ 2012/980438

[5] Zhang XM, Chu YM and Zhang XY (2010). The Hermite-Hadamard type inequality of GA-convex functions and its applications. J. Inequal. Appl. Art. ID 507560, 1-11. https://doi:10.1155/2010/507560

[6] Tariq M (2021). New Hermite-Hadamard type inequalities via p-harmonic exponential type convexity and applications. U. J. Math. Appl. 4(2): 59-69. https://doi .org/10.32323/ujma. 870050

[7] Sahoo SK, Tariq M, Ahmad H, Nasir J, Aydi H and Mukheimer A (2021). New Ostrowski-type fractional integral inequalities via generalized exponential-type convex functions and applications. Symmetry. 13, 1429. https://doi.org/10.3390/sym13081429

[8] Sahoo SK, Ahmad H, Tariq M, Kodamasingh B, Aydi H and De la Sen M (2021). Hermite-Hadamard type inequalities involving $k$-fractional operator for $(\bar{h}, \mathrm{~m})$-convex Functions. Symmetry. 13, 1686. https: //doi.org/10.3390/sym13091686 
[9] Ahmad H, Tariq M, Sahoo SK, Baili J and Cesarano C (2021). New estimations of Hermite-Hadamard type integral inequalities for special functions. Fractal Fract,. 5, 144. https://doi.org/10.3390/ fractalfract 5040144

[10] Omotoyinbo O and Mogbodemu A (2014). Some new Hermite-Hadamard integral inequalities for convex functions. Int. J. Sci. Innovation Tech. 1(1), 1-12.

[11] Tariq M, Nasir J, Sahoo SK, Mallah AA (2021). A note on some Ostrowski type inequalities via generalized exponentially convex function. J. Math. Anal. Model. 2(2): 1-15. https ://doi . org/10.48185/jmam . v2i2. 216

[12] Butt SI, Tariq M, Aslam A, Ahmad H and Nofel, TA (2021). Hermite-Hadamard type inequalities via generalized harmonic exponential convexity. J. Funct. Spaces. 1-12. https : //doi .org/10.1155/2021/5533491

[13] Rafiq A, Mir NA, and Ahmad F (2007). Weighted Chebysev-Ostrowski type inequalities. Applied Math. Mechanics (English Edition). 28(7): 901-906.

[14] Khan MA, Chu YM and Khan TU (2017). Some new inequalities of Hermite-Hadamard type for s-convex functions with applications. Open Math. 151414-1430.

[15] Tariq, M, Sahoo, SK, Nasir, J and Awan, SK (2021). Some Ostrowski type integral inequalities using Hypergeometric Functions. J. Frac. Calc. Nonlinear Sys. 2(1): 24-41.

[16] Aljaaidia TA and Pachpatte D (2021). New generalization of reverse Minkowski's inequality for fractional integral. Adv. Theory. Nonlinear Anal. Appl., 1, 72-81.

[17] Aljaaidia TA and Pachpatte D (2020). Reverse Hermite-Hadamard's inequalities using $\Psi$-fractional integral. Eng. Appl. Sci. Lett.: 1-10.

[18] Aljaaidi TA, Pachpatte DB, Shatanawi W, Abdo MS and Abodayeh K (2021). Generalized proportional fractional integral functional bounds in Minkowski's inequalities Adv. Differ. Equ., 419: 1-17. https: //doi.org/10.1186/s13662-021-03582-8

[19] Alomari M, Darus M and Kirmaci US (2010). Refinements of Hadamard-type inequalities for quasi-convex functions with applications to trapezoidal formula and to special means. Comput. Math. Appl.59: 225-232. https://doi.org/10.1016/j.camwa.2009.08.002

[20] Chen FX and Wu SH (2016). Several complementary inequalities to inequalities of Hermite-Hadamard type for s-convex functions. J. Nonlinear Sci. Appl. 9(2): 705-716.

[21] Tariq M (2020). New Hermite-Hadamard type and some related inequalities via s-type p-convex function. IJSER. 11(12).

[22] Kashuri A and Liko R (2019). Some new Hermite-Hadamard type inequalities and their applications. Stud. Sci. Math. Hung. 56(1): 103-142.

[23] Toader G (1985). Some generalizations of the convexity. Proceedings of The Colloquium On Approximation And Optimization,. Univ. Cluj-Napoca, Cluj-Napoca. 329-338.

[24] Kadakal M and İşcan İ (2020). Exponential type convexity and some related inequalities. J. Inequal. Appl. (1): 1-9. https://doi.org/10.1186/s13660-020-02349-1

[25] Dragomir SS and Agarwal RP (1998). Two inequalities for differentiable mappings and applications to special means of real numbers and to trapezoidal formula. Applied Math. Letters. 11(5): 91-95. https: //doi.org/10.1016/S0893-9659(98)00086-X 